\title{
ASSESSMENT OF COLORECTAL CARCINOMA WITH EMPHASIS ON OUTCOME OF YOUNG AGED PATIENTS- A STUDY OF 62 CASES
}

\author{
Partha Pratim Sinha Roy1, Dwaipayan Samaddar ${ }^{2}$, Gautam Das ${ }^{3}$
}

${ }^{1}$ Specialist Medical Officer, Department of General Surgery, Mal Superspeciality Hospital, Jalpaiguri, West Bengal, India.

${ }_{2}^{2}$ Assistant Professor, Department of General Surgery, North Bengal Medical College and Hospital, West Bengal, India.

3Professor and HOD, Department of General Surgery, North Bengal Medical College and Hospital, West Bengal, India.

\section{BACKGROUND}

\section{ABSTRACT}

Colorectal Carcinoma (CRC) is an upcoming aggressive malignancy, especially among young adult population in recent years. This study emphasises on assessing disease burden and its outcome in young age colorectal carcinoma patients.

The aim of this study is to assess aggressiveness of disease among all CRC patients and to evaluate, post treatment, patient's outcome in all age groups with special emphasis on young age $(<40$ years $)$.

\section{MATERIALS AND METHODS}

A study of patients with features of CRC was conducted in a tertiary care hospital with systematic history documentation, preoperative examinations and post-operative follow-up.

Statistical Analysis- Data was collected and compiled in Excel sheet followed by analysis using software Epi Info and Epi Calc 2000. Data was analysed using Z-test.

Settings and Design- This is a descriptive type of study conducted for a period of two years.

\section{RESULTS}

Maximum CRC patients were in the age group of 30-40 years, mean age 47 years. Rectum was affected more than right and left colon. Ulceroproliferative is the most common morphology observed. Non-mucinous adenocarcinoma is the commonest microscopic variant seen. 16 cases (out of 62) of CRC presented to the emergency department. Patients presented mostly in advanced stage (stage III and IV). 30 of 62 patients received neo-adjuvant therapy. 45 cases went for complete pathological margin negative (R0) resection of carcinoma. Young patients ( $<40$ years) needed more adjuvant therapy and had statistically significant increased incidence of recurrence and metastasis.

\section{CONCLUSION}

Incidence of CRC is remarkable among patients $<40$ years. It is comparatively an aggressive disease with poorer prognosis among young patients.

\section{KEY WORDS}

Colon Cancer, Young Patients, Rectal Cancer, Colorectal Cancer.

HOW TO CITE THIS ARTICLE: Roy PPS, Samaddar D, Das G. Assessment of colorectal carcinoma with emphasis on outcome of young aged patients- a study of 62 cases. J. Evolution Med. Dent. Sci. 2018;7(30):3356-3359, DOI: 10.14260/jemds/2018/757

\section{BACKGROUND}

Colon cancer is the most common cancer of gastrointestinal system in the world.[1] Colorectal carcinoma is the second most common cancer in the western world and also considered to be the third most common cancer in males and the second most common cancer in females.[2,3]

Age is always considered to be a major risk factor for development of colorectal carcinoma. In developed countries, the risk of colon cancer is 1 in 10 males after the $8^{\text {th }}$ decade of life. ${ }^{[4]}$ The national surveillance, epidemiology end result (SEER) data from 1987 to 2006 shows increased incidence of colorectal carcinoma in all 5 years' age groups from 20 years to 49 years of age with maximum increase in 44 to 49 years age group (10.7 per 100,000 in 1988 to 17.9 per 100,000 in 2006).[5] The main concern about young age colorectal

'Financial or Other Competing Interest': None.

Submission 11-06-2018, Peer Review 05-07-2018,

Acceptance 11-07-2018, Published 23-07-2018.

Corresponding Author:

Dr. Partha Pratim Sinha Roy,

15 New Santoshpur First Lane,

Kolkata-700075, West Bengal, India.

E-mail:ppsr88@gmail.com

DOI: $10.14260 /$ jemds $/ 2018 / 757$

\section{(c) $(i)$}

carcinoma is the stigma of poor prognosis attached to it.[6,7] The studies of Duke and Bussey suggested a much higher rate of lymphatic spread in patients less than 40 years of age due to more rapid progression of disease.

The objectives of this study were to assess aggressiveness of disease among all colorectal carcinoma patients and also to evaluate post treatment patient's outcome in all age groups with special emphasis on young age ( $<40$ years).

\section{MATERIALS AND METHODS Study Setting and Design}

A study of patients presenting with features of colorectal carcinoma attending a tertiary care hospital was conducted in the Department of Surgery in collaboration with the Department of Radiotherapy for a period of two years (December 2014 to November 2016). It was a descriptive type of study.

\section{Methodology}

Total number of cases studied was 62. Approval from Ethics Committee was taken. Informed consent from all the patients or guardian of the patient was taken, confidentiality of the findings of the patients was ensured and data was utilised purely for academic purpose. A detailed clinical history was collected from each patient using a preformed data collection 
form. Each patient was examined clinically thoroughly and also investigations were done.

The patients were provided the standard treatment modalities, which include surgical intervention (Curative or palliative) and neo-adjuvant or adjuvant chemotherapy and radiotherapy available in the rural tertiary care hospital. The patient were then followed up for the period of hospital stay and also after 2 weeks post-surgical resection and then at an interval of 3 months for the study period.

The data collected were first charted in Excel sheet. Then analysed by Z-test and p-value was calculated. Categorical variables are expressed as number of patients and percentage of patients and compared using Z-test. The statistical software Epi Info and Epi Calc 2000 had been used for analysis. An alpha level of $5 \%$ had been taken, i.e. if any pvalue was less than 0.05 it had been considered as significant.

\section{RESULTS}

Total 62 cases were studied in the study period of two years. Maximum colorectal carcinoma patients were in the age group of 30-40 years. Mean age was 47 years. Rectum was affected more (26 cases) than right and left colon. Ulceroproliferative appearance was more commonly observed (27 cases) than proliferative (23 cases) or annular (12 cases) growth. Non-mucinous adenocarcinoma (39 cases) was the commonest microscopic variant seen among the colorectal carcinoma. Other microscopic variants were mucinous adenocarcinoma (13 cases) and signet ring adenocarcinoma (10 cases). 16 cases of colorectal carcinoma presented with acute intestinal obstruction to the emergency department, out of them 10 cases were $<40$ years of age. 46 cases presented with the features of colorectal carcinoma in Outpatient Department (OPD) of surgery; out of this 31 cases were $\geq 40$ years of age. Thus, mostly young aged ( $<40$ years) colorectal carcinoma patients presented to the emergency department.

In this study, it had been seen that $24(n=25)$ young aged ( $<40$ years) patients had presented with advanced disease. They were mostly in advanced stage (stage III and IV), which was about $80 \%$ of the total young aged colorectal carcinoma patients $(n=25)$. This study showed a statistically significant more incidence of advanced disease in young aged patients compared to old aged patients ( $\geq 40$ years) (see Table-1).

In this study, 30 patients out of 62 received neoadjuvant therapy. But if analysed critically 16 out of 25 young aged colorectal carcinoma patients received neoadjuvant therapy, which was more than the older population (14 out of 37). And the difference was statistically significant ( $p$-value 0.04338). Among all the patients of colorectal carcinoma, 45 cases out of 62 went for complete pathological margin negative (R0) resection of carcinoma. 9 cases underwent R1 resection and 8 cases underwent $\mathrm{R} 2$ resection.

Most of the patients (54 of 62) received some form of adjuvant therapy, as most patients presented with advanced disease. Among the young aged patients all required adjuvant therapy, but in older group 8 (out of 37) patients did not need any form of adjuvant therapy. The increased incidence of requirement of adjuvant therapy among the young patients ( $<40$ years) was statistically significant ( $p$-value 0.035284 ).

After surgical procedure, patients were followed up for recurrence of colorectal carcinoma. Overall, there were only 6 patients $(n=62)$ who presented with local recurrence. Among them 5 were from the younger age group ( $<40$ years). So we can conclude that there was more incidence of local recurrence among the younger group and it was also statistically significant (see Table-2).

9 out of 62 patients presented with metastasis later. But if considered age-wise, then $6(n=25)$ young patients $(<40$ years) and $3(n=37)$ older patients presented with metastasis. Thus, young age patients $(<40$ years $)$ has statistically significant increased incidence of metastasis (see Table-3).

Various Findings of this Study are also being illustrated with Images (Figure 1)

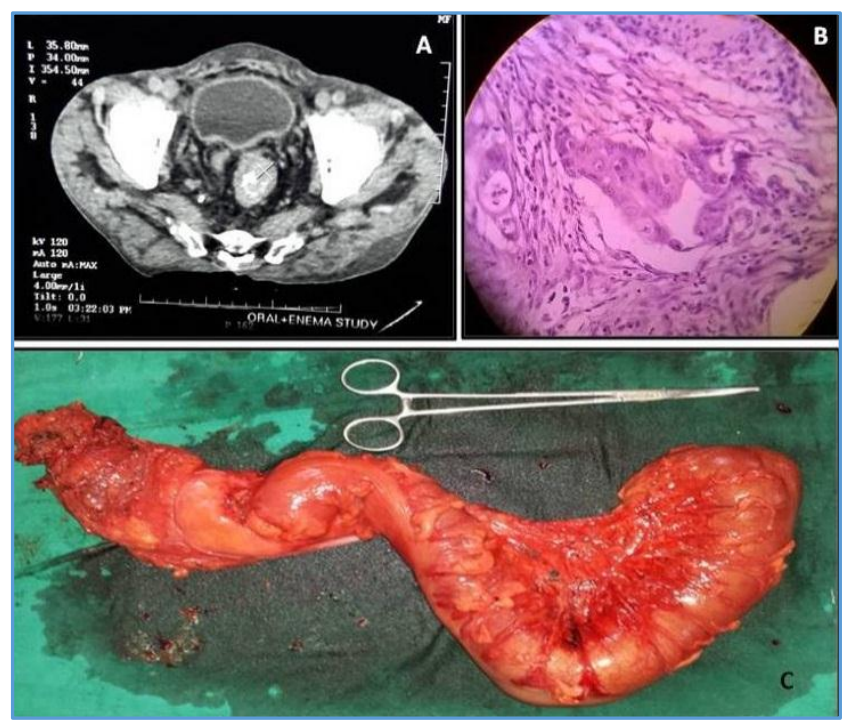

Figure 1. Colorectal Carcinoma. A- Computed Tomography showing Rectal Mass (Marked by Cross). B- Haematoxylin and Eosin Stained Section showing moderately differentiated Colon Carcinoma (400x). C-Resected specimen of Abdominoperineal Resection (APR)

\begin{tabular}{|c|c|c|c|c|}
\hline Age Group & $<\mathbf{4 0}$ yrs. & $\mathbf{\geq 4 0}$ yrs. & Z-test & P-value \\
\hline $\begin{array}{c}\text { Localised disease } \\
\text { (Stage I-II) }\end{array}$ & $\begin{array}{c}1 \\
(4 \%)\end{array}$ & $\begin{array}{c}12 \\
(32.4 \%)\end{array}$ & \multirow{2}{*}{2.698} & 0.00694 \\
\cline { 1 - 3 } $\begin{array}{c}\text { Advanced disease } \\
\text { (Stage III-IV) }\end{array}$ & $\begin{array}{c}24 \\
(96 \%)\end{array}$ & $\begin{array}{c}25 \\
(67.6 \%)\end{array}$ & & \\
& & & & \\
\end{tabular}

Table 1. Stage of Colorectal Carcinoma at Presentation $(n=62)$

\begin{tabular}{|c|c|c|c|c|}
\hline $\begin{array}{c}\text { Local } \\
\text { Recurrence }\end{array}$ & $<\mathbf{4 0}$ yrs. & $\mathbf{2} \mathbf{4 0}$ yrs. & Z-test & P-value \\
\cline { 1 - 3 } Yes & $5(20 \%)$ & $1(3 \%)$ & \multirow{2}{*}{1.88} & 0.02938 \\
\hline No & $20(80 \%)$ & $36(97 \%)$ & & \\
\hline \multicolumn{4}{|c|}{ Table 2 } \\
\hline
\end{tabular}

Table 2. Local Recurrence of Colorectal Carcinoma $(n=62)$

\begin{tabular}{|c|c|c|c|c|}
\hline Metastases & $<\mathbf{4 0}$ yrs. & $\mathbf{2 4 0}$ yrs. & Z-test & P-value \\
\hline Yes & $6(24 \%)$ & $3(8 \%)$ & 1.7425 & 0.04093 \\
\hline No & $19(76 \%)$ & $35(92 \%)$ & & \\
\hline Table 3. Metastasis among Colorectal Carcinoma Patients \\
$(\mathbf{n = 6 2 )}$
\end{tabular}

\section{DISCUSSION}

In this study conducted on the patients attending a tertiary care hospital during two years study period, has shown that 
among 62 colorectal carcinoma patients, 37 people were above the age of 40 years. This is quite in concordance with previous studies, which show more incidences among elderly.[8] Although, colorectal carcinoma is considered to be a disease of the older age group, this study got a significant number of cases in age group below 40 years ( 25 among 62). There was also a peak among the age group 30-40 years. These data justify the previous hypothesis that CRC prevalence in below 40 years' age group is quite significant. Several other studies have also shown that the incidence of CRC cases in older adults has remained stable over the years, but the incidence in young adults (aged 20 to 40 years) has been steadily increasing.[9] The Surveillance, Epidemiology, and End Results (SEER) Registry from 1973 to 1999 indicated that colon and rectal cancers increased by $17 \%$ and $75 \%$, respectively, in persons between 20 and 40 years of age, whereas the rates in those 50 years and older remained stable or declined. ${ }^{[10]}$ CRC is one of the top ten most common cancers amongst those aged 20 to 49 years and for patients in their third and fourth decades CRC is one of the top four most common cancers. ${ }^{[11]}$

Colorectal carcinoma is a broad group comprising of tumour arising from caecum to rectum. But incident of rectal carcinoma is more than other parts of colon according to various previous studies.[2] It was also true for this study, which showed $44 \%$ rectal carcinomas among young age group and $40.5 \%$ among older age group.

Colorectal carcinoma may present with different morphological variants. This study showed a predominance of ulceroproliferative growth among the patients. It was also evident from this study that ulceroproliferative growth was more predominant in younger age group. Adenocarcinoma is the most common histological variant seen in colorectal carcinoma, which was also reflected in this study showing 39 patients $(n=62)$ with adenocarcinoma.[12] But its less frequent variant mucinous adenocarcinoma and signet ring cell adenocarcinoma were also seen. These mucin producing variants are considered to be prognostically worse than nonmucin producing adenocarcinoma.[13,14] But if analysed according to age group, there is comparatively higher incidence of mucin producing tumour (mucinous and signet ring cell adenocarcinoma) among the young aged patients (48\%) than older group (30\%). This may be a factor for poorer outcome among young aged patient.

The patients with colorectal carcinoma may present in emergency with features of acute intestinal obstruction. This study showed that 16 out of 62 patients presented with acute intestinal obstruction, which were managed by diverting stoma followed by elective surgical management after proper workup. But if analysed according to the age group, $40 \%$ of the younger patients presented with acute obstruction compared to $16 \%$ of older age ( $\geq 40$ years) patient, which was also statistically significant. Previous studies has shown that that perioperative mortality following emergency operation was higher compared to elective surgery.[12] So it might be a reason for poorer prognosis amongst young aged colorectal carcinoma patients. The treatment protocol and prognosis of colorectal carcinoma depends on the stage of the disease. In this study, the younger patients $(<40$ years $)$ presented with more advanced malignancy than the older age group. Others have also found the younger population who presents with higher stage disease and less favourable histological variety.[15-19] It is not clear why the younger patients present with more advanced malignancy than their older counterparts. Perhaps this is because of the aggressive nature of the disease amongst the young patients. Another possibility may be that the physicians are less suspicious of malignancy in such young patients leading to delay in advising the appropriate investigation.

Neoadjuvant therapy is a part of the treatment modality used to downstage the disease in rectal carcinoma, so that a curative resection can be possible. In this study, it was observed that 16 of 25 young patients need some form of neoadjuvant therapy before curative surgery can be done, which was significantly more than the older age group (14 out of 37). This can be explained by the fact that young patients presented at a later stage with advanced carcinoma.

Surgery being the mainstay of treatment in colorectal carcinoma, extent of resection was an important prognostic factor. The aim of surgery was to get an R0 resection (pathologically margin negative). In this study, most of the patients (45 of 62) went for R0 resection. Some of the patients underwent neoadjuvant therapy leading to downstaging of the disease followed by surgery in rectal cancers.

Adjuvant treatment was provided to all the patients who presented with advanced disease or in whom R0 resection was not possible. In this study, all the patients (25 out of 25 ) of less than 40 years of age had received post-operative chemotherapy or chemo-radiotherapy, but 8 out of 37 old aged patients did not need any adjuvant therapy.

Recurrence in case of colorectal carcinoma can be divided into local recurrence or distant metastasis. About $30-50 \%$ of colorectal carcinoma may turn up with recurrence after curative resection if they are properly followed up. ${ }^{[20]}$ In this study, there were only 6 local recurrences among 62 cases, which was only $9.6 \%$ of the total study population. But age group wise 5 of the 6 local recurrences were from the younger group. This proves there was a statistically significant increased local recurrence among less than 40 years' age group. This predominance amongst younger age group may be due to the fact that they presented with a higher-grade tumour at a later stage.

As for distant metastasis following curative resection, almost a similar trend was seen. Only 9 out of 62 patients presented with distant recurrence. But if analysed critically, the young populations were worse sufferers as 6 of 9 were $<40$ years of age.

Few studies have shown that prognosis of colorectal carcinoma among young and old age group is comparable if taken stage by stage.[21] This study also hints towards that. But young patients present at a later stage with advanced disease leading to worse prognosis. So the treating physician should always be vigilant in assessing patients with symptoms of colorectal carcinoma.

\section{CONCLUSION}

Incidence of CRC is remarkable among patients $<40$ years. It is comparatively an aggressive disease with poorer prognosis among young patients.

\section{REFERENCES}

[1] Dunn BKM, Rothenberger DA. Colon rectum and anus. In: Brunicardi FC, Anderson DK, Billier RT. eds. 
Schwartz's Principles of Surgery. $10^{\text {th }}$ edn. New York: McGraw-Hill Education 2015: p. 1175-240.

[2] Carlson G, Epstein J. The small and large intestine. In: Williams NS, Bulstrode CJK, O'Connell PR. eds. Bailey \& Love's Short practice of surgery. 26th edn. Boca Raton: Taylor \& Francis Group 2013: p. 1143-80.

[3] Ferlay J, Shin HR, Bray F, et al. GLOBOCAN 2008, cancer incidence and mortality worldwide. IARC Cancer Base no. 10. Vol. (1-2). Lyon, France: International Agency for Research on Cancer 2008.

[4] International Agency for Research on Cancer. 2010. http://globocan.iarc.fr, accessed on January 14, 2018.

[5] Jemal A, Siegel R, Xu J, et al. Cancer statistics, 2010. CA Cancer Journal for Clinicians 2010;60(5):277-300.

[6] Davis DM, Marcet JE, Frattini JC, et al. Is it time to lower the recommended screening age for colorectal cancer? J Am Coll Surg 2011;213(3):352-62.

[7] Fu J, Yang J, Tan Y, et al. Young patients ( $<35$ years old) with colorectal cancer have worse outcomes due to more advanced disease: a 30-year retrospective review. Medicine 2014;93(23):e135.

[8] Chan KK, Dassanayake B, Deen R, et al. Young patients with colorectal cancer have poor survival in the first twenty months after operation and predictable survival in the medium and long-term: analysis of survival and prognostic markers. World Journal of Surgical Oncology 2010;8:82.

[9] Swaminathan R, Shanta V, Ferlay J, et al. Trends in cancer incidence in Chennai city (1982-2006) and state wide predictions of future burden in Tamil Nadu (2007-16). Natl Med J India 2011;24(2):72-7.

[10] O'Connell JB, Maggard MA, Liu JH, et al. Rates of colon and rectal cancers are increasing in young adults. Am Surg 2003;69(10):866-72.

[11] McKay A, Donaleshen J, Helewa RM, et al. Does young age influence the prognosis of colorectal cancer: a population-based analysis? World J Surg Oncol 2014;12(1):370.
[12] Fairley TL, Cardinez CJ, Martin J, et al. Colorectal cancer in U.S. adults younger than 50 years of age, 1998--2001. Cancer 2006;107(Suppl 5):1153-61.

[13] Kaiser AM, Etzioni D, Bert RW. Tumors of Colon. In: Zinner MJ, Ashley SW. eds. Maingot's Abdominal operations. 12th edn. McGraw-Hill 2013: p. 731-66.

[14] O'Connell JB, Maggard MA, Ko CY. Colon cancer survival rates with the new American Joint Committee on Cancer sixth edition staging. J Natl Cancer Inst 2004;96(19):1420-5.

[15] Kanemitsu Y, Kato T, Hirai T, et al. Survival after curative resection for mucinous adenocarcinoma of the colorectum. Dis Colon Rectum 2003;46(2):160-7.

[16] O'Connell JB, Maggard MA, Liu JH, et al. Are survival rates different for young and older patients with rectal cancer? Dis Colon Rectum 2004;47(12):2064-9.

[17] Derwinger K, Kodeda K, Gerjy R. Age aspects of demography, pathology and survival assessment in colorectal cancer. Anticancer Res 2010;30(12):522731.

[18] Ganapathi S, Kumar D, Katsoulas N, et al. Colorectal cancer in the young: trends, characteristics and outcome. Int J Color Dis 2011;26:927-34.

[19] You YN, Dozois EJ, Boardman LA, et al. Young onset rectal cancer: presentation, pattern of care and longterm oncologic outcomes compared to a matched older-onset cohort. Ann Surg Oncol 2011;18(9):246976.

[20] Schellerer VS, Merkel S, Schumann SC, et al. Despite aggressive histopathology survival is not impaired in young patients with colorectal cancer: CRC in patients under 50 years of age. Int J Color Dis 2012;27(1):71-9.

[21] Giovannucci E, Rimm EB, Ascherio A, et al. Alcohol, low-methionine-low-folate diets and risk of colon cancer in men. J Natl Cancer Inst 1995;87(4):265-73. 